INPLASY

PROTOCOL

To cite: Fernandez-Rio et al. What do we know about pedagogical models in physical education and sport so far? An overview of reviews. Inplasy protocol 202130025. doi:

10.37766/inplasy2021.3.0025

Received: 08 March 2021

Published: 08 March 2021

Corresponding author:

Damián Iglesias Gallego

diglesia@unex.es

Author Affiliation:

Teacher Training College.

University of Extremadura.

Spain

Support: None.

Review Stage at time of this submission: Data analysis.

Conflicts of interest:

None declared.

\section{What do we know about pedagogical models in physical education and sport so far? An overview of reviews}

Fernandez-Rio, J1; Iglesias, D².

Review question / Objective: What do we know about pedagogical models in physical education and sport so far? The aim was to provide a broader picture about pedagogical models interventions conducting an overview of reviews.

Condition being studied: Effects promoted by pedagogical models and hybridizations.

Information sources: A comprehensive computerized search of the following electronic databases was performed: Web of Science, Scopus, Psyclnfo, PubMed/Medline, SportDiscus, Eric, Scielo and Taylor \& Francis. We also scanned the reference lists of these papers in order to find other important publications for this overview of reviews.

INPLASY registration number: This protocol was registered with the International Platform of Registered Systematic Review and Meta-Analysis Protocols (INPLASY) on 08 March 2021 and was last updated on 08 March 2021 (registration number INPLASY202130025).

\section{INTRODUCTION}

Review question / Objective: What do we know about pedagogical models in physical education and sport so far? The aim was to provide a broader picture about pedagogical models interventions conducting an overview of reviews.
Rationale: Research into models-based practice in physical education and sport has exponentially increased in the last decades. Several literature reviews on the effectiveness of pedagogical models have been conducted from 2004 to 2020 . These review studies have focused on specific implementations of pedagogical models and even hybridizations. Evidence 
synthesis relevant to Sport Education, Teaching Games for Understanding, Cooperative Learning, Teaching for Personal and Social Responsibility, and hybridizations among pedagogical models have been conducted. Due to the large amount of research that has been conducted, there seems to be a need to synthesize all the evidence found so far in a single updated document.

Condition being studied: Effects promoted by pedagogical models and hybridizations.

\section{METHODS}

Search strategy: The selected reviews were gathered through searching databases: Web of Science, Scopus, Psyclnfo, PubMed/Medline, SportDiscus, Eric, Scielo and Taylor \& Francis. We searched on article title, abstract, and keywords using the following query string: [("models-based practice" OR "pedagogical model" OR "cooperative learning" OR "games-centred approach" OR "teaching games for understanding" OR "sport education" OR "teaching for personal and social responsibility") AND ("physical education" OR "sport") AND ("review")].

Participant or population: Elementary, middle or/and high school students.

Intervention: Pedagogical models and hybridizations.

Comparator: Control, traditional or comparison groups.

Study designs to be included: Systematic reviews and systematic reviews with metaanalysis.

Eligibility criteria: Review studies met the following inclusion criteria: (1) Peerreviewed journal articles (Journal Citation Reports) published and written in English before December 31, 2020, (2) included participants from elementary, middle or/ and high school, (3) conducted in the physical education context or sport, and (4) interventions studies implementing one, several or combined pedagogical models.
Exclusion criteria were: (1) Not review studies, and (2) Not about pedagogical models implementation.

Information sources: A comprehensive computerized search of the following electronic databases was performed: Web of Science, Scopus, Psyclnfo, PubMed/ Medline, SportDiscus, Eric, Scielo and Taylor \& Francis. We also scanned the reference lists of these papers in order to find other important publications for this overview of reviews.

Main outcome(s): Four learning outcomes: cognitive, social, motor and affective domains.

Quality assessment / Risk of bias analysis: The quality assessment of review studies, including risk of bias, was checked using the Assessing the Methodological Quality of Systematic Reviews (AMSTAR-2) tool. Overall rating is calculated from 16 items and allow to classify reviews into four quality level based on weaknesses or flaws: critically low, low, moderate or high.

Strategy of data synthesis: A summary of scope and findings reported of the review studies (author, year, country, participants, interventions, duration, outcomes, conclusions).

Subgroup analysis: No analysis.

Sensitivity analysis: No analysis.

Language: English.

Country(ies) involved: Spain.

Keywords: Models-Based Practice; Pedagogical Models; Physical Education.

Contributions of each author:

Author 1 - Javier Fernandez-Rio - All phases (preparation and writing manuscript).

Email: javier.rio@uniovi.es

Author 2 - Damián Iglesias Gallego - All phases (preparation and writing manuscript).

Email: diglesia@unex.es 